\title{
HDAC6 controls major cell response pathways to cytotoxic accumulation of protein aggregates
}

\author{
Cyril Boyault, ${ }^{1,2,7}$ Yu Zhang, ${ }^{3,7}$ Sabrina Fritah, ${ }^{1,2,7}$ Cécile Caron, ${ }^{1,2}$ Benoit Gilquin, ${ }^{1,2}$ So Hee Kwon, ${ }^{3}$ \\ Carmen Garrido, ${ }^{4,5}$ Tso-Pang Yao, ${ }^{6}$ Claire Vourc' ${ }^{1,},{ }^{1,2}$ Patrick Matthias, ${ }^{3}$ and Saadi Khochbin ${ }^{1,2,8}$ \\ ${ }^{1}$ Institut National de la Santé et de la Recherche Médicale (INSERM), U823, Institut Albert Bonniot, Grenoble F-38706, \\ France; ${ }^{2}$ Université Joseph Fourier, Institut Albert Bonniot, Grenoble F-38700, France; ${ }^{3}$ Friedrich Miescher Institute for \\ Biomedical Research, Novartis Research Foundation, 4058 Basel, Switzerland; ${ }^{4}$ INSERM, U517, Dijon F-21079, France; \\ ${ }^{5}$ Université de Bourgogne, Faculté de Médecine de Dijon, Dijon F-21079, France; ${ }^{6}$ Department of Pharmacology and Cancer \\ Biology, Duke University, Durham, North Carolina 27710, USA
}

A cellular defense mechanism counteracts the deleterious effects of misfolded protein accumulation by eliciting a stress response. The cytoplasmic deacetylase HDAC6 (histone deacetylase 6) was previously shown to be a key element in this response by coordinating the clearance of protein aggregates through aggresome formation and their autophagic degradation. Here, for the first time, we demonstrate that HDAC6 is involved in another crucial cell response to the accumulation of ubiquitinated protein aggregates, and unravel its molecular basis. Indeed, our data show that HDAC6 senses ubiquitinated cellular aggregates and consequently induces the expression of major cellular chaperones by triggering the dissociation of a repressive

HDAC6/HSF1 (heat-shock factor 1)/HSP90 (heat-shock protein 90) complex and a subsequent HSF1 activation. HDAC6 therefore appears as a master regulator of the cell protective response to cytotoxic protein aggregate formation.

[Keywords: HSP25/27; HSP70; acetylation; microtubules; heat shock; p97/VCP]

Supplemental material is available at http://www.genesdev.org.

Received April 10, 2007; revised version accepted July 13, 2007.

HDAC6 (histone deacetylase 6) was first discovered in the mouse as a specific member of a new family of HDACs in higher eukaryotes (Verdel and Khochbin 1999) now known as class II (Yang and Gregoire 2005). Later, studies have shown that HDAC6 is actively maintained in the cytoplasm (Verdel et al. 2000; Bertos et al. 2004), is a major cytoplasmic tubulin-deacetylase (Hubbert et al. 2002; Matsuyama et al. 2002; Zhang et al. 2003), and efficiently binds mono- and polyubiquitin (Seigneurin-Berny et al. 2001; Hook et al. 2002; Boyault et al. 2006).

Furthermore, the identification of an HDAC6-containing complex showed that it associates with two proteins, both involved in the control of the ubiquitin/proteasome system, p97/VCP and UFD3/PLAP /Seigneurin-Berny et al. 2001). Most interestingly, p97/VCP, a chaperone-like AAA ATPase, is essential in the degradation of misfolded proteins by the proteasome (Romisch 2006; Rumpf and Jentsch 2006) and is able to dissociate

\footnotetext{
${ }^{7}$ These authors contributed equally to this work.

${ }^{8}$ Corresponding author.

E-MAIL khochbin@ujf-grenoble.fr; FAX 33-4-76-54-95-95.

Article is online at http://www.genesdev.org/cgi/doi/10.1101/gad.436407.
}

HDAC6-ubiquitin complexes and to modulate the HDAC6-dependent polyubiquitin chain turnover (Boyault et al. 2006). In yeast, Ufd3p, known to interact with the yeast homolog of p97/VCP, Cdc48p /Ghislain et al. 1996), is a modulator of its ubiquitin-dependent functions (Mullally et al. 2006; Rumpf and Jentsch 2006).

HDAC6 therefore has the potential to link protein ubiquitination not only to cellular functions depending on microtubule cytoskeleton, but also to the proteasome-dependent protein degradation. One of these functions has recently been evidenced. Indeed, through its simultaneous interaction with ubiquitin and dynein motors, HDAC6 mediates the clearance of cytotoxic misfolded proteins and favors their accumulation into cellular aggresomes (Kawaguchi et al. 2003). Moreover, recently we were able to show that the cellular HDAC6p97/VCP ratio controls the fate of cellular misfolded ubiquitinated proteins (Boyault et al. 2006). Altogether, these data highlight an essential role for HDAC6 in the management of ubiquitinated cellular protein aggregates.

HDAC6 has also been shown to deacetylate HSP90 and modulate its chaperone activity (Bali et al. 2005; Kovacs et al. 2005; Murphy et al. 2005). HSP90, through 
its diverse actions on its client proteins, appears as a critical modulator of various cell signaling pathways including the control of heat-shock cell response (Zhao and Houry 2005). In fact, HSF1 (heat-shock factor 1), a transcription factor essential in the activation of the heatshock protein-encoding genes (HSPs), is maintained in an inactive form through its association with HSP90, and HSP90-HSF1 dissociation is an obligatory step in the activation of HSF1. Phosphorylation and trimerization of HSF1, following its release from HSP90, then lead to its full activation (Voellmy 2004; Westerheide and Morimoto 2005).

Interestingly, proteasome inhibition and the accumulation of highly ubiquitinated proteins were shown to induce HSF1 activation and the subsequent HSP gene response (Goldberg 2003). Since HDAC6 had already been involved in the protective clearance of misfolded protein aggregates (Kawaguchi et al. 2003; Iwata et al. 2005), we reasoned that it could also signal their presence within the cell to the HSF1 activation machinery, including HSP90. Accordingly, we hypothesized that ubiquitin binding by HDAC6 could act as a sensor of misfolded protein accumulation and elicit HSF1 activation and heat-shock gene response through a mechanism involving HSP90.

Using primary cells and established cell lines isolated from HDAC6-deficient mice $\left(H D A C 6^{y /-}\right)$, or human cells with reduced HDAC6 expression, we were able to confirm this hypothesis and unravel its molecular basis, demonstrating a key role for HDAC6 in controlling HSP genes activation.

Our data show that ubiquitin binding by HDAC6 is a critical event in the dissociation of the repressive HSF1containing complex. In unstressed cells, this complex also contains the HDAC6-interacting chaperone p97/ VCP, which appears to play an important role in the formation of the basal HSP90-HSF1 complex, as well as in the complex dissociation after the accumulation of ubiquitinated misfolded proteins.

Based on these data, we propose that p97/VCP and HDAC6 are at the heart of a very important and yet unknown machinery dedicated to respond to the accumulation of cytotoxic protein aggregates. It first senses abnormal accumulation of polyubiquitinated proteins and triggers HSF1 activation, and then participates in the clearance of these aggregates.

\section{Results}

HDAC6 and its ubiquitin-binding functions are important to induce the expression of HSP genes

Fibroblasts were isolated from HDAC6-deficient $\left(H D A C 6^{y /-}\right)$ or parental wild-type mouse embryos and used to establish $3 \mathrm{~T} 3$ cell lines. A retroviral gene transfer system was then used to reintroduce wild-type HDAC6 (WT) or HDAC6 bearing inactivating mutations in its catalytic domains $\left(\mathrm{HD}^{\mathrm{m}}\right)$ or in its ubiquitin-binding domain, ZnF-UBP $\left(\mathrm{Ub}^{\mathrm{m}}\right)$, in one of the established HDAC6deficient lines.

Figure 1A shows that, as expected, the cellular amount of acetylated tubulin was clearly increased in HDAC6deficient cells (KO), compared with the parental cells. The reintroduction of wild-type HDAC6 restored the basal level of tubulin acetylation (Fig. 1A, WT). The tubulin acetylation levels of the cells expressing the $\mathrm{Ub}^{\mathrm{m}}$ HDAC6 mutant were comparable with those of the cells expressing wild-type HDAC6, while the level of tubulin acetylation in cells expressing the $\mathrm{HD}^{\mathrm{m}}$ HDAC6 mutant was equivalent to that of the KO cells. These cells therefore provide valuable tools to investigate HDAC6 functions related to its catalytic and ubiquitin-binding activities.
A

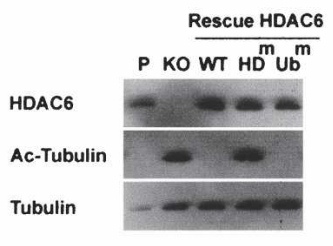

B

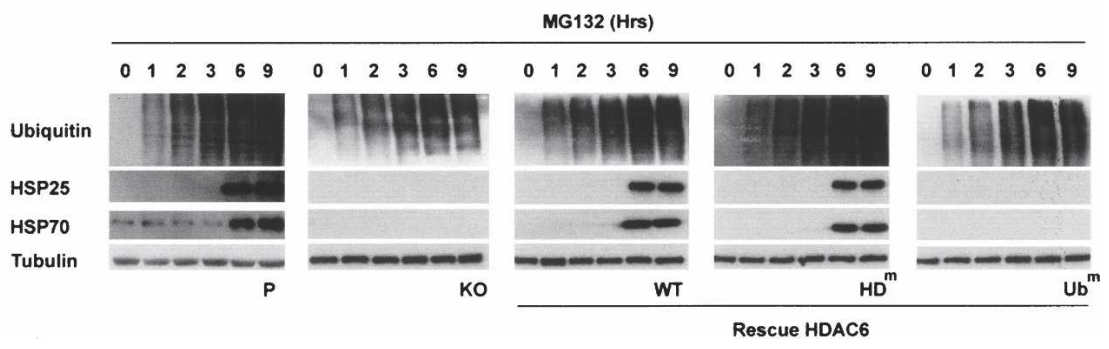

Figure 1. HDAC6 and its ubiquitin-binding activity are required to activate a heatshock cell response to the proteasome inhibition. (A) 3T3 cell lines were established from mouse embryo fibroblasts isolated from parental $(\mathrm{P})$ or HDAC6-deficient $(\mathrm{KO})$ mice. One of the HDAC6-deficient clones was then used to establish new lines re-expressing wild-type HDAC6 (WT) or HDAC6 bearing mutations either in the two catalytic deacetylase domains $\left(\mathrm{HD}^{\mathrm{m}}\right)$ or in the ZnF-UBP ubiquitin-binding domain $\left(\mathrm{Ub}^{\mathrm{m}}\right)$. Equivalent amounts of extracts from these cells were used to compare their levels of HDAC6 expression. The blot was then successively probed with the indicated antibodies. $(B)$ The cell lines described above, treated with $5 \mu \mathrm{g} / \mathrm{mL}$ MG132 for the indicated periods of time, were lysed, and extracts were prepared. Equivalent amounts of extracts were loaded, and ubiquitin, HSP25, HSP70, and tubulin were visualized on Western blots. 
These cell lines were first used to evaluate the role of HDAC6 in controlling the cell response to the induced accumulation of ubiquitinated proteins after a proteasome inhibition by MG132. As shown in Figure 1B, the presence of HDAC6 was absolutely required to observe a significant accumulation of HSP70 and HSP25, $6 \mathrm{~h}$ after the MG132 treatment (cf. KO and parental panels). Interestingly, the re-expression of wild-type HDAC6 completely restored the HSP accumulation in response to proteasome inhibition (Fig. 1B, WT panel). Strikingly, this activity of HDAC6 was dependent on its ability to bind ubiquitin, since the re-expression of the HDAC6 $\mathrm{Ub}^{\mathrm{m}}$ mutant did not restore the HSP gene response after MG132 treatment. This activity of HDAC6 was, however, independent of its deacetylase catalytic activity, since cells expressing this mutant showed an efficient answer to the treatment (Fig. 1B, $\mathrm{HD}^{\mathrm{m}}$ panel).

\section{HDAC6 and its ubiquitin-binding functions mediate HSF1 activation}

It then appeared important to know whether HDAC6 could control the activation of HSF1 after the MG132 treatment.

We first confirmed that HSF1 was, indeed, required to observe an HSP cell response to the MG132 treatment and that proteasome inhibition led to HSF1 activation. Fibroblasts from $\mathrm{HSF}^{-/-}$and the corresponding parental mice (Pirkkala et al. 2000) were treated with MG132, and the activation of HSF1 and accumulation of HSP70 and HSP25 were monitored. Figure 2A shows that in the absence of HSF1, the MG132 treatment was ineffective in inducing an HSP gene response. In the parental cell line, the activation of HSF1 was evidenced by a shift in its migration, visualized on the SDS-PAGE after its phosphorylation.

We then evaluated the role of HDAC6 in HSF1 activation by following HSF1 mobility shift after MG132 treatment in the five cell lines described above. The data obtained evidenced an absolute requirement of HDAC6 for HSF1 activation (Fig. 2B). No HSF1 migration shift was observed in HDAC6-deficient cells after MG132 treatment (Fig. 2B, KO panel). This MG132-dependent HSF1 activation was restored following the re-expression of wild-type HDAC6, but not upon expression of the non-ubiquitin-binding mutant (Fig. 2B, cf. WT and $\mathrm{Ub}^{\mathrm{m}}$ panels). Here again, HSF1 activation was not dependent on HDAC6 catalytic activity (Fig. 2B, panel $\mathrm{HD}^{\mathrm{m}}$ ).

HSF1 activation and subsequent HSP70 expression following MG132 treatment were also monitored in primary cells directly isolated from HDAC6-deficient or parental mice. Figure 2C shows that, as expected, HSF1 activation and HSP70 gene response were not observed in primary HDAC6-deficient cells, while cells isolated from wild-type mice efficiently responded to the treatment. These experiments demonstrated that the observed HDAC6-dependent HSF1 activation in response to the MG132 treatment was not an artifact associated with the establishment of $3 \mathrm{~T} 3$ cells.
Ubiquitin binding by HDAC6 mediates

the dissociation of the repressive

HSP90-HSF1-containing complex

The fact that HDAC6 interacts with and deacetylates HSP90 (Kovacs et al. 2005) points to HSP90 as the best candidate for mediating HDAC6-dependent activation of HSF1 after proteasome inhibition. The five established cell lines described above were left untreated or were treated for $6 \mathrm{~h}$ with MG132. The results in Figure 3A (input panels) confirm that HSF1 activation following MG132 treatment is strictly dependent on the presence of HDAC6 and requires its ubiquitin-binding activity.

Since the dissociation of the HSP90-HSF1 complex was previously shown to be required for HSF1 activation (Zou et al. 1998), we used HSP90 immunoprecipitation to investigate the association of HSF1 and HDAC6 with HSP90 before and after $6 \mathrm{~h}$ of MG132 treatment. After this immunoprecipitation, we also analyzed the state of HSP90 acetylation as well as its association with p97/ $\mathrm{VCP}$; the results obtained regarding these particular points will, however, be considered below, when the involvement of p97/VCP and HSP90 acetylation in the HSP90 complex stability is discussed.

Figure 3A shows that the MG132 treatment induces the dissociation of HSF1 and HDAC6 from HSP90, only in parental cells (Fig. 3A, IP panel, P) and in KO cells expressing wild-type or catalytically dead HDAC6 (Fig. $3 \mathrm{~A}$, IP panel, $\mathrm{WT}$ and $\mathrm{HD}^{\mathrm{m}}$ ). Interestingly, there was no dissociation of HSF1 and HDAC6 when the complex contained an HDAC6 mutant unable to bind ubiquitin (Fig. 3A, IP panel, $\mathrm{Ub}^{\mathrm{m}}$ ). Moreover, these data also show that, in the total absence of HDAC6, the HSP90-HSF1 complex remains stable after the MG132 treatment (Fig. 3A, IP panel, KO).

\section{A mechanism for ubiquitin-dependent complex disassembly and HSF1 activation}

The data obtained thus far strongly suggested that ubiquitin binding by HDAC6 could trigger the HSP90 complex disassembly. To test this hypothesis, the following experiments were performed.

HSP90 was immunoprecipitated from nonstressed 3T3 parental or HDAC6-deficient cells, expressing wild-type or mutated HDAC6, as described above. The immunoprecipitated materials were then incubated with purified pentaubiquitin chains in the presence or absence of ATP, and the proteins remaining associated with the immunocomplexes were analyzed after removal of the released proteins. In the presence of ATP, this in vitro assay perfectly mimicked the in vivo situation. Indeed, pentaubiquitin efficiently dissociated the complex only when wild-type or catalytically inactive HDAC6 was present (Fig. 3B, +ATP panel, columns 11, 13, 14). In the absence of HDAC6, or in the presence of the non-ubiquitin-binding mutant of HDAC6, pentaubiquitin did not dissociate the HSP90-HSF1-containing complexes (Fig. $3 \mathrm{~B}$, columns 12,15$)$.

Interestingly, this experiment also showed that the 


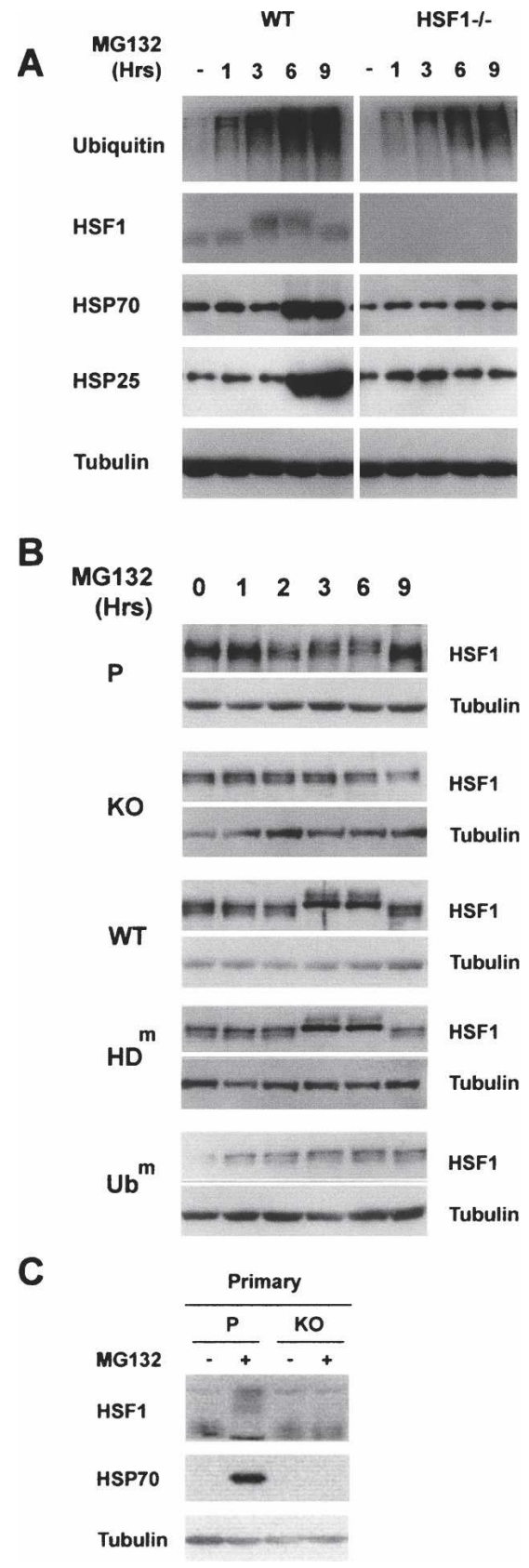

Figure 2. The activation of HSF1 in response to MG132 depends on the integrity of the HDAC6 ZnF-UBP domain. (A) Embryonic MEF cells from parental (WT) and $\mathrm{HSF}^{-/-}$cells were treated with MG132 for the indicated periods of time, and ubiquitin, HSF1, HSP70, HSP25, and tubulin expression was analyzed as above. $(B)$ Extracts from the experiment described in Figure 1B were probed with anti-HSF1 and anti-tubulin antibodies. (C) Primary cells isolated from HDAC6-deficient $(\mathrm{KO})$ and parental $(\mathrm{P})$ mice were treated with MG132 for $6 \mathrm{~h}$ as above $(+)$ or left untreated (-). HSF1 activation and HSP70 accumulation were monitored as shown.

ubiquitin-dependent complex disassembly is an active process, which requires ATP hydrolysis. Indeed, no complex dissociation was observed in the absence of ATP (Fig. 3B, -ATP panel).
A role for $p 97 /$ VCP in the HDAC6-HSP90 complex formation and dissociation

According to the data obtained so far, we hypothesized that, after proteasome inhibition and accumulation of ubiquitinated proteins, ubiquitin binding by HDAC6 could trigger its dissociation from the HSP90-HSF1 complex. However, we also observed that this process required an active participation of HDAC6 (no complex disassembly was detected in the absence of HDAC6) as well as an ATPase activity.

The HDAC6 partner, the AAA ATPase p97/VCP, which we had previously purified from an endogenous HDAC6 complex (Seigneurin-Berny et al. 2001), appeared as a good candidate to mediate the HDAC6-dependent HSP90 complex-dissociating activity. Indeed, we had recently observed that p97/VCP could dissociate the HDAC6-ubiquitin complex (Boyault et al. 2006). Moreover, p97/VCP is well known for its "segregase" activity (Romisch 2006; Rumpf and Jentsch 2006), and, as shown earlier, ubiquitin binding by HDAC6 induces an HDAC6-p97/VCP complex dissociation (SeigneurinBerny et al. 2001).

Accordingly, the presence of $\mathrm{p} 97 / \mathrm{VCP}$ was checked in the HSP90 immunoprecipitated materials. Interestingly, we found that not only was p97/VCP present in a complex with HSP90 but also that the complex formation needed the presence of HDAC6 (Fig. 3A, IP HSP90 panels). Indeed, p97/VCP was not found associated with HSP90 when the latter was immunoprecipitated from HDAC6-deficient cells (Fig. 3A, KO, columns 13, 14).

Based on our previous data (Seigneurin-Berny et al. 2001), we proposed that HDAC6 could mediate the recruitment of $\mathrm{p} 97 / \mathrm{VCP}$ in the repressive HSP90-HSF1 complex. Ubiquitin binding by HDAC6 could then lead to the dissociation of the HDAC6-p97/VCP complex and p97/VCP would use its ATPase activity to dissociate the HSP90-HSF1 complex.

This hypothesis was investigated thanks to an established HDAC6 knocked-down human cell line already used to evidence some important functions of HDAC6 (Kawaguchi et al. 2003; Kovacs et al. 2005). These cells were also of great help because they allowed us to overcome the problem of the very poor transfection efficiency of the established cell lines derived from HDAC6deficient 3T3 cells.

In this cell system, the activation of HSF1 and the subsequent HSP70 accumulation were also dependent on the presence of HDAC6 (Fig. 4A, cf. HDAC6 knockdown cells and wild-type cells; also see Supplementary Fig. 1B).

Therefore, in this independent human cell line, the confirmation of data previously obtained using mouse cell systems on the role of HDAC6 in HSF1 activation and HSP70 accumulation after MG132 treatment allowed us to generalize our conclusions on this particular function of HDAC6.

We then used this same system to investigate the role of $\mathrm{p} 97 / \mathrm{VCP}$ in the HDAC6-dependent HSP response to MG132 treatment. Cells were transfected with spe- 
Figure 3. A treatment with MG132 leads to an HDAC6-dependent dissociation of the repressive HSP90-HSF1 complex. (A) Extracts from the different cell lines described in Figure 1A treated for $6 \mathrm{~h} \mathrm{(+)} \mathrm{with}$ MG132 or untreated (-) were immunoprecipitated with an anti-HSP90 antibody, and the coimmunoprecipitation of HSF1, p97/VCP, and HDAC6 was monitored. The acetylation of the immunoprecipitated HSP90 was also detected using an anti-acetylated lysin antibody. The "input" panel shows the presence of the studied proteins before immunoprecipitation in the extracts. (Right panel) The amount of HSF1 coimmunoprecipitated with HSP90 was estimated by densitometric measurement of HSF1 signals before and after MG132 treatment (shown in the HSF1 lane) and is represented as histograms. The values are expressed as a percent of HSP90-associated HSF1 before the MG132 treatment in each cell line. (B) HSP90 immunocomplexes obtained after the immunoprecipitation of HSP90, as described in $A$, were incubated with $10 \mu \mathrm{g}$ of pentaubiquitin chain in the presence of $2 \mathrm{mM} \mathrm{ATP} / 2 \mathrm{mM} \mathrm{MgCl}{ }_{2}$ or not. After the elimination of the supernatant, the proteins remaining associated with the HSP90 immunocomplex were analyzed by Western blot.

cific anti-p97/VCP or control small interfering RNAs (siRNAs) to monitor HSF1 activation in response to proteasome inhibition. Figure 4B shows that the down-regulation of p97/VCP completely abolished HSF1 activation in response to MG132 treatment (Fig. 4B, WT HDAC6 panel, lane 4; Supplementary Fig. 1B, lane 4).

The role of p97/VCP in the HSP90 complex regulation was further investigated by monitoring the association of HSF1 with HSP90 in unstressed cells treated with control or anti-p97/VCP siRNAs. Interestingly, p97/VCP knockdown led to a significant increase in HSF1-HSP90 association, strongly suggesting that p97/VCP controls the basal level of complex formation.

In MG132-treated cells also, a higher amount of HSF1 remained associated with HSP90, accounting for the less efficient HSF1 activation (Supplementary Fig. 2).

Most interestingly, the knockdown of p97/VCP in unstressed cells also led to a decrease in the recruitment of HDAC6 by HSP90. This observation strongly suggests that $\mathrm{p} 97 / \mathrm{VCP}$ is also required to form the basal HSP90 HDAC6 complex in unstressed cells. These data are in perfect agreement with our previous work (Boyault et al. 2006), showing that p97/VCP was, indeed, required to "extract" ubiquitin-bound HDAC6 and, in other words, to "recycle" HDAC6.

We can now propose that p97/VCP is necessary to re-
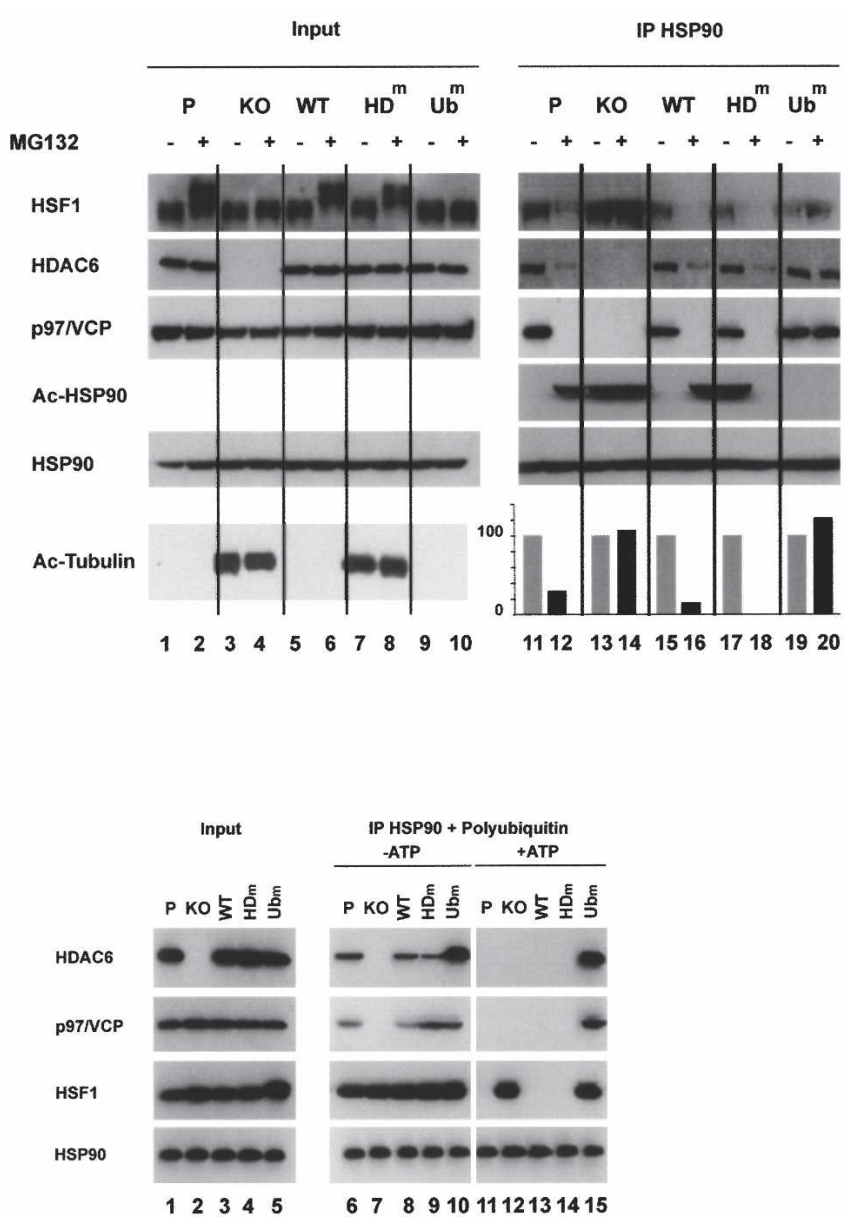

cover HDAC6 from its interaction with other cellular components, and that, in unstressed cells, it is the HDAC6-p97/VCP complex that is recruited to the HSP90 complex to form the "basal complex."

In the presence of a decreased amount of p97/VCP, HDAC6 remains in its sites of action and cannot be used to form the basal HSP90 complex.

Altogether these data allowed to complete our model and to describe in detail the molecular events leading to the HSP90-HSF1 complex formation in unstressed cells and to its ubiquitin-dependent dissociation after the accumulation of ubiquitinated cellular aggregates.

\section{Is there a role for HDAC6-dependent HSP9O deacetylation in HSF1 activation in response to MG132 treatment?}

We also monitored the state of HSP90 acetylation in the five established cell lines described above before and after MG132 treatment. HSP90 acetylation was detected after its immunoprecipitation (shown in Fig. 3A) by an anti-acetylated lysine antibody. As expected, HSP90 was found acetylated in the $\mathrm{KO}$ cells as well as in cells expressing the catalytically dead HDAC6, confirming that HDAC6 is a major HSP90 deacetylase (Fig. 3A, IP HSP90 panel, lane AcHSP90, columns 13, 17). Figure 3A also 
A

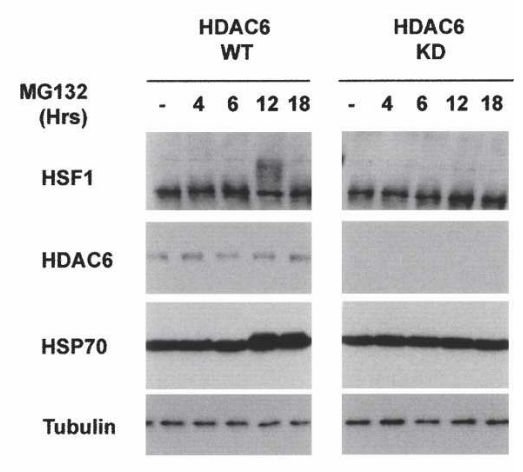

B

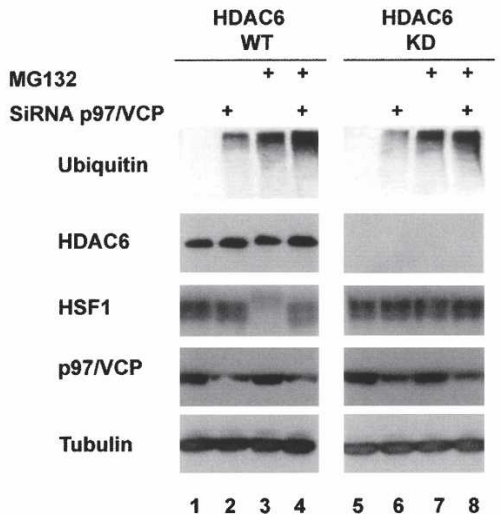

Figure 4. p97/VCP mediates the HDAC6-dependent HSF1 activation. (A) Human HEK cells stably expressing an antiHDAC6 shRNA (KD) or the control cell lines (WT) (Kawaguchi

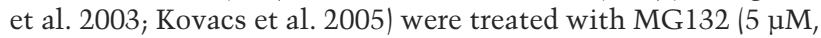
up to $18 \mathrm{~h}$ ), and extracts were prepared after indicated periods of time. The corresponding blots were then probed with the antiHSF1, anti-HSP70, and anti-tubulin antibodies. (B) HDAC6 knocked-down HEK (KD) and the control (WT) cell lines were treated with control siRNA or siRNA designed against p97/ $\mathrm{VCP}$, and cells were treated with $5 \mu \mathrm{M} \mathrm{MG} 132$ during $6 \mathrm{~h}$ as indicated. Extracts were prepared and probed with antibodies against HSF1, ubiquitin, p97/VCP, and tubulin.

shows that, in parental or in $\mathrm{KO}$ cells re-expressing wildtype HDAC6, proteasome inhibition leads to a clear increase in HSP90 acetylation, which very well correlates with the HDAC6-HSP90 complex dissociation observed in these cells (columns 12, 16).

In cells expressing the $\mathrm{Ub}^{\mathrm{m}}$ HDAC6 mutant, HSP90 remained underacetylated before and after MG132 treatment in perfect agreement with the inability of this HDAC6 mutant to be released from HSP90 after MG132 treatment (Fig. 3A, columns 19, 20).

However, an intriguing situation was observed in cells expressing the catalytically dead HDAC6 mutant $\left(\mathrm{HD}^{\mathrm{m}}\right)$. In these cells, as expected, HSP90 was found acetylated before MG132 treatment (Fig. 3A, column 17), but curiously, the acetylation signal disappeared $6 \mathrm{~h}$ after the treatment (Fig. 3A, column 18) in the total absence of active HDAC6. This could be due to the intervention of another HDAC, which could be activated only when the $\mathrm{HD}^{\mathrm{m}}$ HDAC6 mutant is released. This putative HDAC does not, however, show any tubulin-deacetylase activity, since, in these cells, tubulin remained highly acetylated after the MG132 treatment (Fig. 3A, input panel, lanes 7,8).

These data show that no clear relationship can be established between HSP90 acetylation and HSF1 activation and HSP gene response. This conclusion is also supported by the fact that a catalytically dead mutant of HDAC6 is as active as the wild-type protein in inducing HSP gene activation in response to a treatment with MG132 (Figs. 1B, 2B, 3A), as well as in HSP90 complex disassembly (Fig. 3A).

\section{The impairment of proteasome activity is essential to trigger the HDAC6-dependent cell response}

In all the experiments presented here, MG132 was used as a means to inhibit the proteasome activity and to induce the HDAC6-dependent cell response. MG132 may, however, have uncontrolled effects on various cellular functions. In order to convincingly show that the impairment of proteasome function elicits the HDAC6mediated response, we used siRNA against PAC1, a factor recently shown to be critical in the assembly of the active 20S proteasome (Hirano et al. 2005). Down-regulation of PAC1 in control cells, as expected, leads to a significant accumulation of ubiquitinated proteins after $32 \mathrm{~h}$ of siRNA treatment, indicative of a proteasome impairment in both cell lines (Fig. 5A). In contrast to wildtype cells, in HDAC6 knockdown cells, despite the accumulation of ubiquitinated proteins after PAC1 downregulation, no HSF1 activation or heat-shock gene response was observed. These data confirmed our conclusions on the role of HDAC6 in sensing the proteasome dysfunction.

Involvement of HDAC6 in protecting cells against the toxic effects of misfolded protein accumulation

The data presented here point to HDAC6 as an essential cell survival element after the accumulation of misfolded ubiquitinated proteins. In order to confirm this hypothesis, we monitored cell apoptosis after their treatment with MG132. Figure 6 shows that sublethal doses of MG132 become significantly toxic in the absence of HDAC6 or when cells express a mutated version of HDAC6.

Our data also show that cells expressing a catalytically dead HDAC6 are also hypersensitive to an MG132 treatment, despite the fact that they are still capable of inducing the HSP gene response. This could be explained by the involvement of HDAC6 catalytic activity in the formation of protective aggresomes and their degradation by autophagy, through a yet-unknown mechanism (Kawaguchi et al. 2003; Iwata et al. 2005). The most dramatic effect, as expected, was observed in cells expressing the non-ubiquitin-binding mutant of HDAC6. 
A

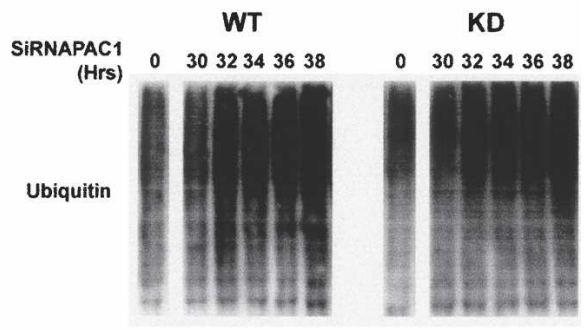

B

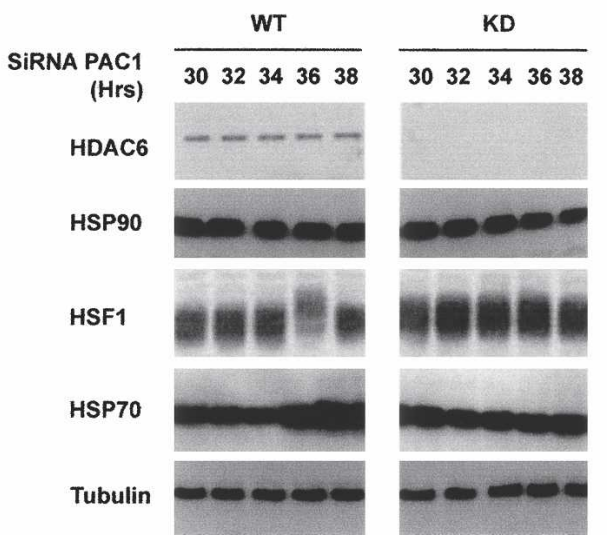

Figure 5. Impairment of the proteasome activity leads to an HDAC6-dependent activation of HSF1. Human HEK KD (HDAC6 knockdown) and control cells were treated with an anti-PAC1 siRNA for the indicated periods of time, and the accumulation of polyubiquitinated proteins $(A)$ and activation of HSF1 and HSP70 $(B)$ accumulation were monitored. The level of general protein ubiquitination and HSP70 did not change before $30 \mathrm{~h}$ of transfection and was comparable with that of nontransfected cells (" 0 " in $A$ and not shown).

\section{Discussion}

The proteasome/ubiquitin system clears the important mass of cellular misfolded proteins, which are continuously generated. These nonfunctional proteins can arise by mutations, excess in subunit synthesis, and various denaturing stresses (Goldberg 2003). Moreover, an important portion of newly synthesized proteins consists of misfolded products, which also contribute to increase the mass of nonfunctional cellular proteins (Schubert et al. 2000). These defective proteins have a tendency to assemble into cytotoxic aggregates (Sherman and Goldberg 2001), and different cellular defense mechanisms operate to counteract their deleterious effects.

First, a cellular clearance mechanism has been proposed to take charge of the aggregates and concentrate them into a single juxta-nuclear inclusion body named the "aggresome" (Johnston et al. 1998). This process is thought to reduce the cytotoxic effects of scattered cellular protein aggregates (Kopito 2000). HDAC6 was recently shown to be a key player in this clearance mechanism by functioning as an adaptor that, through its ubiq- uitin-binding activity, links aggregated protein cargos to the dynein motor, thus mediating their transport to the aggresome (Kawaguchi et al. 2003).

The appearance of the aggresome or inclusion body in cells, however, induces dramatic impairments of many cellular functions including a global inactivation of the ubiquitin/proteasome system (Bennett et al. 2005), which collectively paralyze vital cellular activities and induce cell death. Interestingly, HDAC6 was also shown to mediate the recruitment of the autophagic machinery to aggresomes, thereby further decreasing the cytotoxic effects of these aggregates (Iwata et al. 2005).

Another cellular mechanism helps facing protein aggregate toxicity by activating a heat-shock gene response circuit, leading to the accumulation of major cellular chaperones (Kawazoe et al. 1998; Lee and Goldberg 1998; Mathew et al. 1998, 2001; Kim et al. 1999). These chaperones decrease aggregate toxicity and facilitate their resorption (Goldberg 2003). They indeed possess the potential to prevent aggregate formation either by helping protein refolding or by assisting their delivery to the ubiquitin/proteasome degradation system (Hohfeld et al. 2001).

Here we show that HDAC6 is also a major player in the latter mechanism, and is involved in signaling the abnormal accumulation of ubiquitinated proteins to the stress-response transcriptional machinery. The use of HDAC6-deficient cells re-expressing wild-type or mutated HDAC6 allowed us to precisely dissect the underlying mechanism.

In the absence of cellular aggregates like HSF1, both HDAC6 and p97/VCP remain in a dormant complex with HSP90. We show here that $\mathrm{p} 97 / \mathrm{VCP}$ is a critical player in the formation of the basal HSP90-HDAC6 complex in unstressed cells. Indeed, p97/VCP was hypothesized here to be required to recycle HDAC6 involved in various cellular activities in order to form the basal HSP90 complex in unstressed cells.

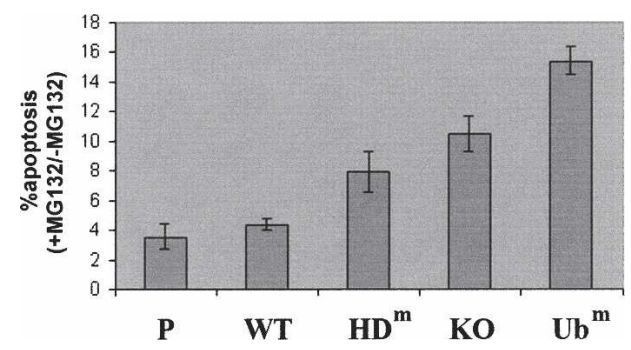

Figure 6. HDAC6 and its ubiquitin-binding activity protect cells against the cytotoxic effects of proteasome dysfunction. The indicated cell lines (as in Fig. 1) were left untreated or were treated with $2 \mu \mathrm{M}$ MG132 for $16 \mathrm{~h}$, and the apoptotic cells were visualized by the detection of the active caspase 3 and FACS analysis. The proportion of apoptotic cells in each population was measured and was presented as a ratio of treated over untreated cells. The histograms represent three independent experiments, and the variations around the mean values are indicated. The mean values for the percentage of apoptotic cells in the untreated population are as follows: parental cells, 0.3 ; wild type (WT), 0.27; $\mathrm{HD}^{\mathrm{m}}, 0.25 ; \mathrm{KO}, 0.28 ; \mathrm{Ub}^{\mathrm{m}}, 0.21$. 
The accumulation of ubiquitinated proteins following the impairment of the proteasome activity is sensed by HDAC6, via its ubiquitin-binding activity. HDAC6ubiquitin interaction is the initiator of a chain of events evidenced here, inducing the dissociation of the HDAC6-p97/VCP complex. According to our model, the whole mechanism of this complex dissociation is built around this ubiquitin-dependent HDAC6-p97/VCP dissociation. Indeed, we had previously shown that ubiquitin binding by HDAC6 led to a HDAC6-p97VCP complex dissociation (Seigneurin-Berny et al. 2001). More recently, we were able to show that free p97/VCP can also extract ubiquitin-bound HDAC6 and reinduce the complex formation (Boyault et al. 2006). Ubiquitin is therefore a strong regulator of HDAC6-p97/VCP interaction. Hence, our model proposes that, after the ubiquitin-dependent dissociation of HDAC6-p97/VCP, the latter uses its segregase activity to dissociate the repressive HSP90 complex, eventually leading to the release of HSF1. Alternatively, it is also possible that p97/VCP, directly or indirectly, stimulates HSP90's own ATPase activity or positively regulates the activity of its various cochaperones.

The sensing of ubiquitinated protein aggregates by HDAC6 therefore elicits three critical components of the cell response to their accumulation: HSF1, which will induce the accumulation of major HSPs; HDAC6, which will mediate protein aggregate clearance as reported previously (Kawaguchi et al. 2003; Iwata et al. 2005); and p97/VCP, which is a well-known regulator of misfolded protein degradation (Woodman 2003; Rumpf and Jentsch 2006). Indeed, p97/VCP has a major role in controlling the degradation by the ubiquitin/proteasome system of a variety of endoplasmic reticulum-associated degradation (ERAD) substrates (Romisch 2006; Rumpf and Jentsch 2006). According to our recent data (Boyault et al. 2006), p97/VCP may also play an important role in the post-stress recovery stages by extracting the ubiquitin-bound HDAC6. The reformation of p97/VCPHDAC6 would then provide a platform for the reassociation of HSP90 and HSF1 and re-establishment of the basal nonstressed state.

During these investigations, no evidence was found for a role of HSP90 acetylation in HSF1-HSP90 complex stability or in the process of HSF1 activation in response to the impairment of proteasome activity. In agreement with previously published data (Kawaguchi et al. 2003; Iwata et al. 2005), a role of HDAC6 catalytic activity was, however, found in protecting cells against the toxic effect of MG132. This probably reflects a need for the deacetylase activity of HDAC6 after its release from the HSP90 complex, in the transport and clearance of cellular aggregates (Kawaguchi et al. 2003; Iwata et al. 2005). In these experiments, cells expressing the non-ubiquitinbinding mutant of HDAC6 also showed a higher sensitivity to MG132 treatment compared with that of HDAC6 KO cells. This could be due to a dominant-negative effect of this mutant, which may titer out some cellular factors important in the cytoprotective cell response to MG132 treatment.
It is also important to mention that we did not observe a clear role for HDAC6 in HSF1 activation in response to heat shock (Supplementary Fig. 1A). It can therefore be concluded that the obligatory step of HSF1-HSP90 dissociation, in the stress-dependent HSF1 activation, is controlled by distinct mechanisms depending on the nature of the stressful stimuli. Accordingly, the siRNAmediated knockdown of p97/VCP while completely abolishing the MG132-dependent activation of HSF1 had no effect on the heat-shock-dependent activation of the protein (Supplementary Fig. 1B). In the case of heat shock, the release of HSF1 may be controlled by stimuli that occur earlier than, or probably not directly depending on, the accumulation of misfolded ubiquitinated proteins.

Interestingly, during these investigations, we have also observed that treating HDAC6-expressing cells with MG132 for $6 \mathrm{~h}$ leads to a twofold accumulation of HSF1-encoding mRNA (data not shown). This observation points to a new mechanism, which allows a supply of fresh HSF1 in addition to the HDAC6-dependent mobilization of the HSP90-stored pool of HSF1. The fact that HDAC6 was absolutely required for HSF1 activation after $6 \mathrm{~h}$ of MG132 treatment suggests that most of the HSF1 present in the cells at this time point comes from the HSP90-associated stock. The neosynthesized pool of HSF1 is likely to be directly activated and hence does not require a HDAC6-dependent eventi.e., HSP90 complex dissociation-prior to activation. It is therefore expected that events leading to an extensive accumulation of newly synthesized HSF1 would induce an HDAC6-independent response of heat-shock genes.

The mechanism described here places HDAC6 in the heart of a response system specifically activated after the impairment of proteasome activity, when misfolded protein aggregates accumulate. The beauty of the model is that, in the repressive HSP90 complex, HDAC6 and p97/ VCP first serve as a trigger and a responsive dissociating spring, respectively, and then, after their release, both individually behave as actors of the aggregate management, while HSF1 follows its activation route to elicit additional help by inducing a massive synthesis of chaperones.

\section{Materials and methods}

\section{Cell lines}

Mice with an invalidated HDAC6 gene were generated (Zhang et al. 2003; Y. Zhang and P. Matthias, unpubl.), and mouse embryo fibroblasts were isolated from embryonic day 13.5 (E13.5) embryos. Sex genotyping was used to select only male embryos. HDAC6 knockout and wild-type lines were further identified by PCR genotyping and Western blots. 3T3 cell lines were established following a standard 3T3 protocol. The cells were plated at the density of $3 \times 10^{5}$ cells per 5 -cm plate and split every $3 \mathrm{~d}$ for $\sim 20$ passages.

Wild-type and mutant HDAC6 cDNAs (Verdel et al. 2000; Seigneurin-Berny et al. 2001) were cloned into a pMSCV.EGFP vector. The retrovirus was made from Pheonix cells following a 
standard protocol. After the infection, HDAC6-deficient 3T3 cells were kept in culture for $2 \mathrm{wk}$, and single GFP-positive cells were sorted into 96-well plates. The positive clones were identified by Western blot and PCR.

$\mathrm{HSF}^{-/-}$and parental cells have been described already (Christians et al. 1997). HEK cells stably expressing anti-HDAC6 and control short hairpin RNAs (shRNAs) have been described previously (Kawaguchi et al. 2003; Kovacs et al. 2005).

\section{Plasmids, siRNAs, and treatments}

Mouse HDAC6 cDNAs, wild type or harboring inactivating mutations in both catalytic domains or in the ubiquitin-binding ZnF-UBP domain, were described and characterized previously (Verdel et al. 2000; Seigneurin-Berny et al. 2001). These cDNAs were used to generate all the HDAC6 pMSCV.EGFP vectors employed to establish the stable cell lines described above.

Anti-PAC1 and anti-p97/VCP siRNAs were designed as described previously (Wojcik et al. 2004; Hirano et al. 2005) and were purchased from Eurogentec. HEK cells were seeded in sixwell plates at $10^{5}$ cells per well, $24 \mathrm{~h}$ before the siRNA treatment. In each experiment, HAT1 siRNA was used as a control. siRNA transfections were performed using oligofectamine reagent (Invitrogen) according to the manufacturer's instructions.

Proteasome inhibitor MG132 was purchased from Calbiochem (474790). MG132 was used at $5 \mu \mathrm{M}$ except for FACS analysis, where cells were treated during $24 \mathrm{~h}$ with $2 \mu \mathrm{M}$ MG132.

\section{Antibodies, immunoprecipitation, and FACS analysis}

The antibodies used are as follows: p97/VCP antibody was a gift of Dr. N.K. Tonks (Cold Spring Harbor, NY). Anti-HDAC6 was described previously (Seigneurin-Berny et al. 2001). Anti-ubiquitin, anti-HSF1, and anti-tubulin were purchased from Santa Cruz Biotechnology (SC-8017), Stressgen (SPA-901), and Sigma (T5168), respectively. Anti-acetylated $\alpha$ Tubulin, anti-HSP70, anti-HSP25, anti-HSP27, and anti-HSP90 were from Sigma (T6793), Abcam (3A3 ab5439), and Stressgen (SPA-801, SPA803, and SPA-830), respectively. Anti-acetylated lysines were purchased from Cell Signaling (9441).

Immunoprecipitations were performed on cytosolic extracts. Cytosolic extracts were prepared as described (Seigneurin-Berny et al. 2001) using a buffer of the following composition: $50 \mathrm{mM}$ HEPES (pH 7.4), $60 \mathrm{mM} \mathrm{KCl}, 15 \mathrm{mM} \mathrm{NaCl}, 0.65 \mathrm{mM}$ Spermidine, 0.34 M Saccharose, $2 \mathrm{mM}$ EDTA, $0.5 \mathrm{mM}$ EGTA, $0.5 \mathrm{mM}$ DTT, $0.05 \%$ Triton, anti-protease cocktail (Complete Tabs; Roche), anti-phosphatase cocktail 1 (Sigma P2850, 1/100e), BGlycerophosphate (Sigma G6251; 25 mM), NaF (Sigma S7920; 5 $\mathrm{mM}$ ), and Trichostatine A (Sigma; $100 \mathrm{ng} / \mathrm{mL}$ ). On cytosolic extract, $0.5 \%$ CHAPS, $50 \mathrm{mM} \mathrm{MgCl}_{2}$, and $5 \mathrm{mM} \mathrm{CaCl}_{2}$ were added.

For immunoprecipitation of HSP90, $200 \mu \mathrm{g}$ of a cytosoluble cell extract were incubated with $2 \mu \mathrm{g}$ of HSP90 antibody for 12 $\mathrm{h}$, and captured on protein $\mathrm{G}$ sepharose 4 fast-flow beads (Amersham Biosciences) for $2 \mathrm{~h}$. After washing, beads were boiled in loading buffer and analyzed by Western blot. Anti-phosphatase cocktail 1, B-Glycerophosphate (Sigma G6251; $25 \mathrm{mM}$ ), NaF (Sigma S7920; $5 \mathrm{mM}$ ), Trichostatine A (Sigma; $100 \mathrm{ng} / \mathrm{mL}$ ), Complete Tabs (Roche), 0.5\% CHAPS, $50 \mathrm{mM} \mathrm{MgCl}_{2}$, and $5 \mathrm{mM}$ $\mathrm{CaCl}_{2}$ were used during each step of HSP90 immunoprecipitation.

Ubiquitin-dependent immunocomplex dissociation assays were performed by incubating HSP90 immunocomplexes obtained after HSP90 immunoprecipitation with $10 \mu \mathrm{g}$ of pentaubiquitin (ubiquitin ${ }_{5}^{+1}$ from Affinity) in a buffer with $50 \mathrm{mM}$
HEPES (pH 7.4), $60 \mathrm{mM} \mathrm{KCl,} 15 \mathrm{mM} \mathrm{NaCl}, 0.65 \mathrm{mM}$ Spermidine, $0.34 \mathrm{M}$ Saccharose, $2 \mathrm{mM}$ EDTA, $0.5 \mathrm{mM}$ EGTA, $0.5 \mathrm{mM}$ DTT, $0.05 \%$ Triton, anti-protease coktail (Complete Tabs; Roche), anti-phosphatase cocktail 1 (Sigma P2850; 1/100e), BGlycerophosphate (Sigma G6251; 25 mM), NaF (Sigma S7920; 5 $\mathrm{mM}$ ), and Trichostatine A (Sigma; $100 \mathrm{ng} / \mathrm{mL}$ ), containing 2 $\mathrm{mM}$ ATP and $2 \mathrm{mM} \mathrm{MgCl}$ or not for $2 \mathrm{~h}$ at room temperature. After centrifugation and washing the beads, proteins present in the immunocomplexes were analyzed by Western blot.

Apoptotic cells were visualized by FACS after the detection of active caspase 3 using a BD Pharmingen kit, according to the vendor's instructions.

\section{Acknowledgments}

We gratefully acknowledge the association "vaincre la mucoviscidose" for supporting C.B.'s Ph.D. fellowship for 4 years. S.K.'s laboratory was supported by the EpiMed and EpiPro (CLARA/INCa) as well as ARECA (ARC) programs. We gratefully acknowledge the expert assistance of Sandrine Benitski in tissue culture work and Drs. Sophie Rousseaux and Caroline Jolly for critical reading of the manuscript. The work in P.M.'s laboratory was supported by the Novartis Research Foundation. The work in C.V.'s laboratory was supported by the EpiPro (CLARA/INCa) and ARECA (ARC) programs, as well as by ARC (grant 3449). S.F. was supported by the French ministry of research for 3 years and ARC for 1 year.

\section{References}

Bali, P., Pranpat, M., Bradner, J., Balasis, M., Fiskus, W., Guo, F., Rocha, K., Kumaraswamy, S., Boyapalle, S., Atadja, P., et al. 2005. Inhibition of histone deacetylase 6 acetylates and disrupts the chaperone function of heat shock protein 90: A novel basis for antileukemia activity of histone deacetylase inhibitors. J. Biol. Chem. 280: 26729-26734.

Bennett, E.J., Bence, N.F., Jayakumar, R., and Kopito, R.R. 2005. Global impairment of the ubiquitin-proteasome system by nuclear or cytoplasmic protein aggregates precedes inclusion body formation. Mol. Cell 17: 351-365.

Bertos, N.R., Gilquin, B., Chan, G.K., Yen, T.J., Khochbin, S., and Yang, X.J. 2004. Role of the tetradecapeptide repeat domain of human histone deacetylase 6 in cytoplasmic retention. J. Biol. Chem. 279: 48246-48254.

Boyault, C., Gilquin, B., Zhang, Y., Rybin, V., Garman, E., Meyer-Klaucke, W., Matthias, P., Muller, C.W., and Khochbin, S. 2006. HDAC6-p97/VCP controlled polyubiquitin chain turnover. EMBO J. 25: 3357-3366.

Christians, E., Michel, E., Adenot, P., Mezger, V., Rallu, M., Morange, M., and Renard, J.P. 1997. Evidence for the involvement of mouse heat shock factor 1 in the atypical expression of the HSP70.1 heat shock gene during mouse zygotic genome activation. Mol. Cell. Biol. 17: 778-788.

Ghislain, M., Dohmen, R.J., Levy, F., and Varshavsky, A. 1996. Cdc48p interacts with Ufd3p, a WD repeat protein required for ubiquitin-mediated proteolysis in Saccharomyces cerevisiae. EMBO J. 15: 4884-4899.

Goldberg, A.L. 2003. Protein degradation and protection against misfolded or damaged proteins. Nature 426: 895-899.

Hirano, Y., Hendil, K.B., Yashiroda, H., Iemura, S., Nagane, R., Hioki, Y., Natsume, T., Tanaka, K., and Murata, S. 2005. A heterodimeric complex that promotes the assembly of mammalian 20S proteasomes. Nature 437: 1381-1385.

Hohfeld, J., Cyr, D.M., and Patterson, C. 2001. From the cradle 
to the grave: Molecular chaperones that may choose between folding and degradation. EMBO Rep. 2: 885-890.

Hook, S.S., Orian, A., Cowley, S.M., and Eisenman, R.N. 2002. Histone deacetylase 6 binds polyubiquitin through its zinc finger (PAZ domain) and copurifies with deubiquitinating enzymes. Proc. Nat1. Acad. Sci. 99: 13425-13430.

Hubbert, C., Guardiola, A., Shao, R., Kawaguchi, Y., Ito, A., Nixon, A., Yoshida, M., Wang, X.F., and Yao, T.P. 2002. HDAC6 is a microtubule-associated deacetylase. Nature 417: 455-458.

Iwata, A., Riley, B.E., Johnston, J.A., and Kopito, R.R. 2005. HDAC6 and microtubules are required for autophagic degradation of aggregated huntingtin. J. Biol. Chem. 280: 4028240292.

Johnston, J.A., Ward, C.L., and Kopito, R.R. 1998. Aggresomes: A cellular response to misfolded proteins. J. Cell Biol. 143: $1883-1898$

Kawaguchi, Y., Kovacs, J.J., McLaurin, A., Vance, J.M., Ito, A., and Yao, T.P. 2003. The deacetylase HDAC6 regulates aggresome formation and cell viability in response to misfolded protein stress. Cell 115: 727-738.

Kawazoe, Y., Nakai, A., Tanabe, M., and Nagata, K. 1998. Proteasome inhibition leads to the activation of all members of the heat-shock-factor family. Eur. J. Biochem. 255: 356-362.

Kim, D., Kim, S.H., and Li, G.C. 1999. Proteasome inhibitors MG132 and lactacystin hyperphosphorylate HSF1 and induce hsp70 and hsp27 expression. Biochem. Biophys. Res. Commun. 254: 264-268.

Kopito, R.R. 2000. Aggresomes, inclusion bodies and protein aggregation. Trends Cell Biol. 10: 524-530.

Kovacs, J.J., Murphy, P.J., Gaillard, S., Zhao, X., Wu, J.T., Nicchitta, C.V., Yoshida, M., Toft, D.O., Pratt, W.B., and Yao, T.P. 2005. HDAC6 regulates Hsp90 acetylation and chaperone-dependent activation of glucocorticoid receptor. Mol. Cell 18: 601-607.

Lee, D.H. and Goldberg, A.L. 1998. Proteasome inhibitors: Valuable new tools for cell biologists. Trends Cell Biol. 8: 397403.

Mathew, A., Mathur, S.K., and Morimoto, R.I. 1998. Heat shock response and protein degradation: Regulation of HSF2 by the ubiquitin-proteasome pathway. Mol. Cell. Biol. 18: 50915098.

Mathew, A., Mathur, S.K., Jolly, C., Fox, S.G., Kim, S., and Morimoto, R.I. 2001. Stress-specific activation and repression of heat shock factors 1 and 2. Mol. Cell. Biol. 21: 71637171.

Matsuyama, A., Shimazu, T., Sumida, Y., Saito, A., Yoshimatsu, Y., Seigneurin-Berny, D., Osada, H., Komatsu, Y., Nishino, N., Khochbin, S., et al. 2002. In vivo destabilization of dynamic microtubules by HDAC6-mediated deacetylation. EMBO J. 21: 6820-6831.

Mullally, J.E., Chernova, T., and Wilkinson, K.D. 2006. Doal is a Cdc48 adapter that possesses a novel ubiquitin binding domain. Mol. Cell. Biol. 26: 822-830.

Murphy, P.J., Morishima, Y., Kovacs, J.J., Yao, T.P., and Pratt, W.B. 2005. Regulation of the dynamics of hsp90 action on the glucocorticoid receptor by acetylation/deacetylation of the chaperone. J. Biol. Chem. 280: 33792-33799.

Pirkkala, L., Alastalo, T.P., Zuo, X., Benjamin, I.J., and Sistonen, L. 2000. Disruption of heat shock factor 1 reveals an essential role in the ubiquitin proteolytic pathway. Mol. Cell. Biol. 20: 2670-2675.

Romisch, K. 2006. Cdc48p is UBX-linked to ER ubiquitin ligases. Trends Biochem. Sci. 31: 24-25.

Rumpf, S. and Jentsch, S. 2006. Functional division of substrate processing cofactors of the ubiquitin-selective Cdc48 chap- erone. Mol. Cell 21: 261-269.

Schubert, U., Ott, D.E., Chertova, E.N., Welker, R., Tessmer, U., Princiotta, M.F., Bennink, J.R., Krausslich, H.G., and Yewdell, J.W. 2000. Proteasome inhibition interferes with gag polyprotein processing, release, and maturation of HIV-1 and HIV-2. Proc. Nat1. Acad. Sci. 97: 13057-13062.

Seigneurin-Berny, D., Verdel, A., Curtet, S., Lemercier, C., Garin, J., Rousseaux, S., and Khochbin, S. 2001. Identification of components of the murine histone deacetylase 6 complex: Link between acetylation and ubiquitination signaling pathways. Mol. Cell. Biol. 21: 8035-8044.

Sherman, M.Y. and Goldberg, A.L. 2001. Cellular defenses against unfolded proteins: A cell biologist thinks about neurodegenerative diseases. Neuron 29: 15-32.

Verdel, A. and Khochbin, S. 1999. Identification of a new family of higher eukaryotic histone deacetylases. Coordinate expression of differentiation-dependent chromatin modifiers. J. Biol. Chem. 274: 2440-2445.

Verdel, A., Curtet, S., Brocard, M.P., Rousseaux, S., Lemercier, C., Yoshida, M., and Khochbin, S. 2000. Active maintenance of mHDA2/mHDAC6 histone-deacetylase in the cytoplasm. Curr. Biol. 10: 747-749.

Voellmy, R. 2004. On mechanisms that control heat shock transcription factor activity in metazoan cells. Cell Stress Chaperones 9: 122-133.

Westerheide, S.D. and Morimoto, R.I. 2005. Heat shock response modulators as therapeutic tools for diseases of protein conformation. J. Biol. Chem. 280: 33097-33100.

Wojcik, C., Yano, M., and DeMartino, G.N. 2004. RNA interference of valosin-containing protein $(\mathrm{VCP} / \mathrm{p} 97)$ reveals multiple cellular roles linked to ubiquitin/proteasome-dependent proteolysis. J. Cell Sci. 117: 281-292.

Woodman, P.G. 2003. p97, a protein coping with multiple identities. J. Cell Sci. 116: 4283-4290.

Yang, X.J. and Gregoire, S. 2005. Class II histone deacetylases: From sequence to function, regulation, and clinical implication. Mol. Cell. Biol. 25: 2873-2884.

Zhang, Y., Li, N., Caron, C., Matthias, G., Hess, D., Khochbin, S., and Matthias, P. 2003. HDAC-6 interacts with and deacetylates tubulin and microtubules in vivo. $E M B O I .22$ : 1168-1179.

Zhao, R. and Houry, W.A. 2005. Hsp90: A chaperone for protein folding and gene regulation. Biochem. Cell Biol. 83: 703710.

Zou, J., Guo, Y., Guettouche, T., Smith, D.F., and Voellmy, R. 1998. Repression of heat shock transcription factor HSF1 activation by HSP90 (HSP90 complex) that forms a stresssensitive complex with HSF1. Cell 94: 471-480. 


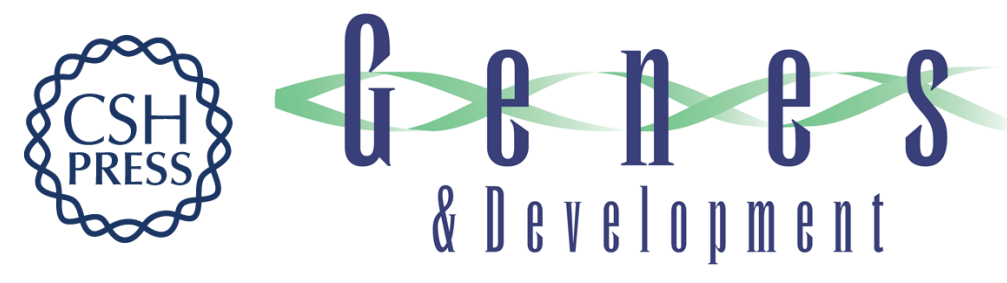

\section{HDAC6 controls major cell response pathways to cytotoxic accumulation of protein aggregates}

Cyril Boyault, Yu Zhang, Sabrina Fritah, et al.

Genes Dev. 2007, 21:

Access the most recent version at doi:10.1101/gad.436407

Supplemental http://genesdev.cshlp.org/content/suppl/2007/08/16/21.17.2172.DC1
Material

References This article cites 40 articles, 20 of which can be accessed free at:

http://genesdev.cshlp.org/content/21/17/2172.full.html\#ref-list-1

License

Email Alerting Receive free email alerts when new articles cite this article - sign up in the box at the top

Service

right corner of the article or click here.

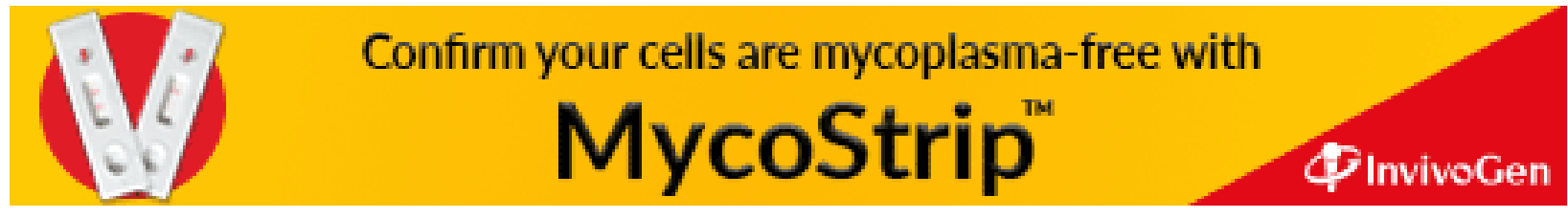

